DOI 10.34883/PI.2021.7.3.005

УДК: 616.155.392.2-036.11-053.2/.81:577.21]-07-037

Корзик А.В., Вшивкова О.С.

Республиканский научно-практический центр детской онкологии, гематологии

и иммунологии, Минск, Беларусь

Korzik A., Vshyukova V.

Belarusian Research Center for Pediatric Oncology, Hematology and Immunology, Minsk, Belarus

\title{
BCR-ABL1-подобный острый
}

лимфобластный лейкоз: от биологии

к перспективным методам терапии

\author{
BCR-ABL1-Like Acute Lymphoblastic Leukemia: \\ from Biology to Promising Therapies
}

Резюме

BCR-ABL1-подобный острый лимфобластный лейкоз (ОЛЛ) может быть охарактеризован как лейкоз из B-линейных предшественников со сходным с BCR-ABL1-позитивным ОЛЛ профилем экспрессии генов при отсутствии транслокации t(9;22)/BCR-ABL1, а также наличием множественных аберраций, активирующих передачу сигналов киназных или цитокиновых рецепторов [1, 2].

Впервые BCR-ABL1-подобный ОлЛ был выделен как отдельный подтип лейкоза в 2009 г. 2 независимыми группами исследователей в результате математического анализа данных, полученных при профилировании экспрессии генов (GEP). Детской онкологической группой (COG) совместно с детской исследовательской больницей Св. Иуды (SJCRH) под руководством Ch. Mullighan был использован метод предсказательного анализа микрочипов (prediction analysis of microarrays, PAM) для сравнения экспрессии 257 зондов в образцах BCR-ABL1позитивного и BCR-ABL1-негативного ОЛЛ. В результате анализа была обнаружена категория образцов, схожих по экспрессии генов с BCR-ABL1-позитивным ОЛЛ, но не несущих этой транслокации, названная в последующем Ph-подобным ОлЛ. Параллельно группа исследователей из Нидерландов (медицинский центр Эразма Роттердамского (DCOG)) под руководством M. den Boer использовала иерархический кластерный анализ (hierarchical clustering, НC) данных по экспрессии 110 зондов. Задачей исследователей было классифицировать педиатрических пациентов на уже известные молекулярные подгруппы ОЛЛ, в числе которых была выделена группа BCR-ABL1-подобного ОЛЛ. Результаты, полученные 2 исследовательскими группами, коррелируют между собой, однако различаются методологией анализа профиля экспрессии генов, составом выборки пациентов, а панели микрочипов сопоставимы только по 9 зондам, что объясняет расхождения в частоте встречаемости BCR-ABL1-подобного ОЛЛ в первых публикациях [2-4]. Различия, которые наблюдаются при сравнении 2 ключевых исследований, подчеркивают гетерогенность случаев BCR-ABL1-подобного ОЛЛ. Тем не менее исследования M. den Boer и Ch. Mullighan стали основой для более глубокого изучения особенностей нового подтипа ОЛЛ. Позже было установлено крайне неблагоприятное течение заболевания, в результате чего в 2016 г. Всемирная организация здравоохранения (ВО3) признала и включила BCR-ABL1-подобный ОЛЛ как предварительную единицу в классификацию В-линейных острых лимфобластных лейкозов/лимфом, подтверждая его клиническую 
важность. Тем не менее, ввиду гетерогенности BCR-ABL1-подобного ОЛЛ, его дифференциальная диагностика и стратификация пациентов в клинической практике до сих пор остаются трудной, но очень важной задачей $[5,6]$. В данном обзоре описаны результаты ключевых исследований BCR-ABL1-подобного ОЛЛ, перечислены генетические особенности и основные подходы к диагностике нового подтипа лейкоза, а также перспективные терапевтические стратегии.

Ключевые слова: BCR-ABL1-подобный ОЛЛ, $\mathrm{Ph}$-подобный ОЛЛ, острый лимфобластный лейкоз.

\section{Abstract}

BCR-ABL1-like acute lymphoblastic leukemia (ALL) can be characterized as leukemia from B-line precursors with the gene expression profile similar to BCR-ABL1-positive ALL in the absence of $\mathrm{t}(9 ; 22) / \mathrm{BCR}-\mathrm{ABL} 1$ translocation, as well as the presence of multiple aberrations that activate the signaling of kinase or cytokine receptors [1, 2].

For the first time, BCR-ABL1-like ALL was isolated as a separate subtype of leukemia in 2009 by two independent research groups as a result of mathematical analysis of the data obtained from the gene expression profiling (GEP). Children's Oncology Group (COG) in conjunction with St. Jude Children's Research Hospital (SJCRH) led by Ch. Mullighan used the prediction analysis of microarrays (PAM) to compare the expression of 257 probes in BCR-ABL1-positive and BCR-ABL1negative ALL samples. The analysis revealed a category of samples similar in gene expression to BCR-ABL1-positive ALL, but not carrying this translocation, which was subsequently named "Ph-like ALL". In parallel, a group of researchers from the Netherlands (Erasmus Medical Center of Rotterdam (DCOG)) led by M. den Boer used the hierarchical clustering $(\mathrm{HC})$ analysis of 110 probe expression data. The task of the researchers was to classify pediatric patients into already known molecular subgroups of ALL, among which the BCR-ABL1-like ALL was identified. The results obtained by the two research groups correlate with each other, but they differ in the methodology for analyzing the gene expression profile, the composition of the patient sample; and the microarray panels are comparable only for nine probes, which explains the discrepancies in the frequency of occurrence of BCR-ABL1-like ALL in the first publications $[2,3,4]$. The differences observed when comparing the two key studies highlight the heterogeneity of BCR-ABL1-like ALL. However, the studies by M. den Boer and Ch. Mullighan became the base for a deeper study of the features of the new ALL subtype. Later, an extremely unfavorable course of the disease was revealed. As a result, in 2016, the World Health Organization (WHO) recognized and included BCR-ABL1-like ALL as a preliminary unit in the classification of B-linear acute lymphoblastic leukemias/lymphomas, confirming its clinical importance. Nevertheless, due to the heterogeneity of BCR-ABL1-like ALL, its differential diagnosis and patient stratification in clinical practice is still a difficult but necessary task [5, 6]. This review describes the results of key studies of BCR-ABL1-like ALL, lists the genetic characteristics and main approaches to the diagnosis of a new subtype of leukemia, as well as the promising therapeutic strategies.

Keywords: BCR-ABL1-like ALL, Ph-like ALL, acute lymphoblastic leukemia.

\section{Распространенность BCR-ABL1-подобного ОЛЛ}

По данным различных исследований, BCR-ABL1-подобный ОЛЛ включает до 10\% пациентов детского возраста, определяемых ВО3 в группу стандартного риска, и до 15\% пациентов высокого риска [7]. В отличие от BCR-ABL1 ОЛЛ, частота встречаемости которого увеличивается с возрастом пациентов, частота BCR-ABL1-подобного ОЛЛ, по-видимому, достигает максимума у подростков и молодых взрослых (16-39 лет) и 
снижается до $10 \%$ у пациентов в возрасте от 40 до 85 лет (табл. 1) [8, 9]. Частота встречаемости у детей до 16 лет составляет 13-15\% от всех случаев В-линейных ОЛЛ. Тем не менее, по оценкам различных ретроспективных исследований, частота может варьировать в зависимости

\section{Таблица 1}

Частота встречаемости BCR-ABL1-подобного ОлЛ у детей, подростков и взрослых согласно данным различных исследований

\begin{tabular}{|c|c|c|c|c|}
\hline $\begin{array}{l}\text { Автор (исследова- } \\
\text { ние, год) }\end{array}$ & $\begin{array}{l}\text { Характеристика } \\
\text { выборки: возраст } \\
\text { (кол-во пациентов) }\end{array}$ & $\begin{array}{l}\text { Кол-во случа- } \\
\text { ев BCR-ABL1- } \\
\text { подобного ОлЛ }\end{array}$ & $\begin{array}{l}\text { Частота } \\
\text { BCR-ABL1- } \\
\text { подобного ОлЛ, \% }\end{array}$ & $\begin{array}{l}\text { Метод диагно- } \\
\text { стики }\end{array}$ \\
\hline $\begin{array}{l}\text { Herold и соавт. } \\
\text { (GMALL, 2014) [9] }\end{array}$ & $\begin{array}{l}16-20(n=26) \\
21-39(n=68) \\
40-55(n=45) \\
55-84(n=67)\end{array}$ & $\begin{array}{l}5 \\
12 \\
4 \\
5\end{array}$ & $\begin{array}{l}19 \\
18 \\
9 \\
7\end{array}$ & GEP, FISH \\
\hline $\begin{array}{l}\text { Jain и соавт. } \\
\text { (MDACC, 2016) [10] }\end{array}$ & $\begin{array}{l}15-39(n=80) \\
40-84(n=68)\end{array}$ & $\begin{array}{l}33 \\
16\end{array}$ & $\begin{array}{l}42 \\
24\end{array}$ & GEP, FISH \\
\hline $\begin{array}{l}\text { Boer и соавт. } \\
\text { (Dutch-Belgium } \\
\text { HOVON, 2015) [11] }\end{array}$ & $\begin{array}{l}16-20(n=24) \\
21-39(n=48) \\
40-71(n=55)\end{array}$ & $\begin{array}{l}24 \\
48 \\
55\end{array}$ & $\begin{array}{l}25 \\
19 \\
11\end{array}$ & GEP \\
\hline $\begin{array}{l}\text { Roberts и co- } \\
\text { aвт. (Cancer and } \\
\text { Leukemia Group B, } \\
\text { 2017) [12] }\end{array}$ & $\begin{array}{l}21-39(n=344) \\
40-59(n=304) \\
60-86(n=150)\end{array}$ & $\begin{array}{l}96 \\
62 \\
36\end{array}$ & $\begin{array}{l}28 \\
20 \\
24\end{array}$ & $\begin{array}{l}\text { GEP; } \\
\text { секвенирование } \\
\text { транскриптома }\end{array}$ \\
\hline $\begin{array}{l}\text { Roberts и соавт. } \\
\text { (European Genome } \\
\text { Phenome archive, } \\
\text { 2014) [13] }\end{array}$ & $\begin{array}{l}1-15(n=853) \\
16-20(n=372) \\
21-39(n=168)\end{array}$ & $\begin{array}{l}108 \\
77 \\
46\end{array}$ & $\begin{array}{l}13 \\
21 \\
27\end{array}$ & $\begin{array}{l}\text { NGS; } \\
\text { GEP; цитогенетиче- } \\
\text { ское исследование }\end{array}$ \\
\hline $\begin{array}{l}\text { Loh и соавт. (P9906/ } \\
\text { AALL0232, 2013) } \\
{[14]}\end{array}$ & $1-31(n=572)$ & 81 & 14 & GEP \\
\hline $\begin{array}{l}\text { Reshmi и со- } \\
\text { авт. (AALL1131/ } \\
\text { AALL0932, 2017) } \\
{[15]}\end{array}$ & $1-31(n=1389)$ & 284 & 20 & GEP \\
\hline
\end{tabular}

Примечания: GEP - профилирование экспрессии генов; FISH - флуоресцентная гибридизация in situ; NGS - секвенирование нового поколения.

Table 1

The incidence of BCR-ABL1-like ALL in children, adolescents and adults according to various studies

\begin{tabular}{|c|c|c|c|c|}
\hline Author (study, year) & $\begin{array}{l}\text { Sample characteristic: } \\
\text { age (number of patients) }\end{array}$ & $\begin{array}{l}\text { Number of cases of the } \\
\text { BCR-ABL1-like ALL }\end{array}$ & $\begin{array}{l}\text { Frequency of } \\
\text { BCR-ABL1-like ALL, \% }\end{array}$ & Diagnostic method \\
\hline Herold et al. (GMALL, 2014) [9] & $\begin{array}{l}16-20(n=26) \\
21-39(n=68) \\
40-55(n=45) \\
55-84(n=67)\end{array}$ & $\begin{array}{l}5 \\
12 \\
4 \\
5\end{array}$ & $\begin{array}{l}19 \\
18 \\
9 \\
7 \\
\end{array}$ & GEP, FISH \\
\hline Jain et al. (MDACC, 2016) [10] & $\begin{array}{l}15-39(n=80) \\
40-84(n=68)\end{array}$ & $\begin{array}{l}33 \\
16\end{array}$ & $\begin{array}{l}42 \\
24\end{array}$ & GEP, FISH \\
\hline $\begin{array}{l}\text { Roberts et al. (Cancer and Leukemia } \\
\text { Group B, 2017) [12] }\end{array}$ & $\begin{array}{l}21-39(n=344) \\
40-59(n=304) \\
60-86(n=150)\end{array}$ & $\begin{array}{l}96 \\
62 \\
36\end{array}$ & $\begin{array}{l}28 \\
20 \\
24\end{array}$ & $\begin{array}{l}\text { GEP; } \\
\text { transcriptome sequencing }\end{array}$ \\
\hline $\begin{array}{l}\text { Roberts et al. (European Genome } \\
\text { Phenome archive, 2014) [13] }\end{array}$ & $\begin{array}{l}1-15(n=853) \\
16-20(n=372) \\
21-39(n=168)\end{array}$ & $\begin{array}{l}108 \\
77 \\
46\end{array}$ & $\begin{array}{l}13 \\
21 \\
27\end{array}$ & $\begin{array}{l}\text { NGS; } \\
\text { GEP; cytogenetic study }\end{array}$ \\
\hline
\end{tabular}

Notes: GEP - gene expression profiling; FISH - fluorescence in situ hybridization; NGS - next generation sequencing. 
от групп риска, этнического состав выборки и разнообразия методологий, используемых для диагностики BCR-ABL1-подобного ОЛЛ [7].

BCR-ABL1-подобный ОЛЛ чаще встречается у пациентов мужского пола во всех возрастных группах, при этом соотношение мужчин и женщин составляет 1,5:1 среди детей и возрастает до 4:1 среди молодых взрослых $[8,12]$. Однако не все исследования подтверждают эту зависимость [12]. В одном европейском исследовании, напротив, была продемонстрирована значительно более высокая доля женщин с BCRABL1-подобным ОЛЛ, хотя не было никаких значимых различий в возрасте или показателях инициального лейкоцитоза [16].

\section{Клинические особенности и прогноз}

Выявление пациентов с BCR-ABL1-подобным ОЛЛ при постановке диагноза имеет решающее значение для уточнения стратификации риска и оптимизации терапевтических стратегий. С момента выделения BCR-ABL1-подобного ОЛЛ в отдельную подгруппу B-линейных лейкозов он неизменно классифицируется как подтип высокого риска, поскольку ассоциирован с тяжелым течением и плохим прогнозом, в частности более высокой частотой рецидивов. В подавляющем большинстве исследований продемонстрированы худшие результаты выживаемости без событий (EFS) и общей выживаемости (OS) у пациентов с BCRABL1-подобным ОЛЛ по сравнению с BCR-ABL1-негативными случаями. BCR-ABL1-подобный профиль экспрессии сопровождается гиперлейкоцитозом, высоким уровнем минимальной остаточной болезни (МОБ) после индукционной терапии, в сравнении с пациентами с BCR-ABL1негативным ОЛЛ $[8,17]$.

\section{Таблица 2}

Выживаемость при BCR-ABL1-подобном ОЛЛ: сравнительная характеристика наиболее значимых исследований с 2009 по 2020 г.

\begin{tabular}{|c|c|c|}
\hline $\begin{array}{l}\text { Исследование } \\
\text { (страна, руководитель; год) }\end{array}$ & $\begin{array}{l}\text { Характеристика выборки: } \\
\text { кол-во пациентов, возраст, группа риска }\end{array}$ & $\begin{array}{l}\text { Выживаемость, } \\
\text { \% }\end{array}$ \\
\hline $\begin{array}{l}\text { COG AALL0232 (США - Канада, } \\
\text { Harvey-Mullighan; 2013) [14] }\end{array}$ & $\begin{array}{l}\mathrm{n}=572 \\
1-30 \text { лет } \\
\text { группа высокого риска }\end{array}$ & 62,6 (EFS) \\
\hline $\begin{array}{l}\text { St. Jude Total XV (США, 2007-2010; } \\
\text { K.G. Roberts) [18] }\end{array}$ & $\begin{array}{l}\mathrm{n}=344 \\
1-18 \text { лет } \\
\text { все группы риска }\end{array}$ & $\begin{array}{l}90,0 \text { (EFS) } \\
92,5 \text { (OS) }\end{array}$ \\
\hline $\begin{array}{l}\text { COALL92/97 (Германия, Den Boer; } \\
\text { 2009) [20] }\end{array}$ & $\begin{array}{l}\mathrm{n}=154 \\
0-18 \text { лет } \\
\text { все группы риска }\end{array}$ & 59,5 (DFS) \\
\hline $\begin{array}{l}\text { HOVON (Дания - Бельгия, J.M. Boer; } \\
\text { 2015) [11] }\end{array}$ & $\begin{array}{l}\mathrm{n}=127 \\
16-71 \text { год } \\
\text { все группы риска }\end{array}$ & 25 (EFS) \\
\hline GMALL (Германия, T. Herold; 2017) [19] & $\begin{array}{l}\mathrm{n}=207 \\
16-84 \text { года } \\
\text { все группы риска }\end{array}$ & 26 (DFS) \\
\hline $\begin{array}{l}\text { Multiple US (США, K.G. Roberts; 2016) } \\
\text { [12] }\end{array}$ & $\begin{array}{l}\mathrm{n}=344 \\
21-39 \text { лет } \\
\text { все группы риска }\end{array}$ & 22,5 (EFS) \\
\hline
\end{tabular}

Примечание: DFS - disease-free survival, выживаемость без признаков заболевания (в данном случае лимфобластного лейкоза). 
Table 2

Survival in BCR-ABL1-like ALL: comparative characteristics of the most significant studies from 2009 to 2020

\begin{tabular}{|l|l|l|}
\hline $\begin{array}{l}\text { Study } \\
\text { (country, supervisor; year) }\end{array}$ & Sample characteristic: number of patients, age, risk group & Survival, \% \\
\hline $\begin{array}{l}\text { COG AALL0232 (USA - Canada, Harvey-Mullighan; } \\
\text { 2013) [14] }\end{array}$ & $\begin{array}{l}n=572 \\
1-30 \text { years } \\
\text { high risk group }\end{array}$ & 62,6 (EFS) \\
\hline $\begin{array}{l}\text { St. Jude Total XV (USA, 2007-2010; K.G. Roberts) } \\
\text { [18] }\end{array}$ & $\begin{array}{l}n=344 \\
1-18 \text { years } \\
\text { all risk groups }\end{array}$ & $\begin{array}{l}90,0 \text { (EFS) } \\
92,5 \text { (OS) }\end{array}$ \\
\hline COALL92/97 (Germany, Den Boer; 2009) [20] & $\begin{array}{l}n=154 \\
0-18 \text { years } \\
\text { all risk groups }\end{array}$ & 59,5 (DFS) \\
\hline HOVON (Denmark - Belgium, J.M. Boer; 2015) [11] & $\begin{array}{l}n=127 \\
16-71 \text { years } \\
\text { all risk groups }\end{array}$ & 25 (EFS) \\
\hline GMALL (Germany, T. Herold; 2017) [19] & $\begin{array}{l}n=207 \\
16-84 \text { years } \\
\text { all risk groups }\end{array}$ & 26 (DFS) \\
\hline Multiple US (USA, K.G. Roberts; 2016) [12] & $\begin{array}{l}n=344 \\
21-39 \text { years } \\
\text { all risk groups }\end{array}$ & 22,5 (EFS) \\
\hline
\end{tabular}

Note: DFS - disease-free survival (without lymphoblastic leukemia).

Прогноз пациентов с BCR-ABL1-подобным ОЛЛ зависит от возраста: по данным некоторых исследований, у взрослых показатели выживаемости хуже, чем у детей и подростков: 5-летняя EFS - 24,1\% против 41,0 и $58,2 \%$ соответственно, а 5-летняя OS - 25,8\% против 72,8 и $65,8 \%$ соответственно [7, 15]. Результаты оценки выживаемости при BCR-ABL1подобном ОЛЛ, полученные в наиболее значимых исследованиях, представлены в табл. 2.

В одном из исследований продемонстрированы эквивалентные результаты МОБ-ассоциированной терапии у детей с BCR-ABL1-подобным и BCR-ABL1-негативным ОЛЛ [18]. В другом педиатрическом исследовании статус МОБ имел бо́льшую прогностическую ценность для общей выживаемости, чем BCR-ABL1-подобный профиль лейкоза, что подчеркивает важность строгого мониторинга МОБ при ОЛЛ [19]. Напротив, исследование, проведенное с участием взрослых пациентов с BCR-ABL1подобным ОЛЛ, не выявило взаимосвязи уровня МОБ с ухудшением результатов долгосрочной выживаемости [10]. Таким образом, высокая частота рецидивов у пациентов с BCR-ABL1-подобным ОЛЛ, особенно у взрослых, не обязательно коррелирует с длительным сохранением МОБ, что ставит вопрос о проведении аллогенной трансплантации стволовых клеток в первой полной ремиссии независимо от уровня МОБ.

\section{Перспективы терапии BCR-ABL1-подобного олл}

Поскольку большинство случаев BCR-ABL1-подобного ОЛЛ сопровождается генетическими изменениями генов киназ и/или цитокиновых рецепторов, областью активных исследований и клинических испытаний стало использование ингибиторов тирозинкиназ у первичных пациентов и у пациентов с плохим ранним ответом для улучшения клинических исходов [1]. В настоящее время зарегистрировано 6 клинических исследований по применению ингибиторов тирозинкиназ при BCRABL1-подобном ОЛЛ, однако лишь 1 из них завершено. Это исследование II фазы по определению максимально переносимой дозы и частоты 
ответа при лечении пациентов с BCR-ABL1-подобным ОЛЛ руксолитинибом (ингибитор янус-киназ) в сравнении с дазатинибом (ингибитор тирозинкиназы BCR-ABL1, а также киназ SRC-семейства, c-KIT, EPHA2, PDGFR $\beta$ и BTK), однако результаты исследования не опубликованы.

В контексте применения ингибиторов тирозинкиназ в настоящее время изучаются 2 основных пути передачи сигналов: каскады $A B L$ и JAK-STAT. Перестройки генов семейства ABL (известно около 12 слияний генов) встречаются в среднем у 17\% детей с BCR-ABL1-подобным ОЛЛ, 9\% подростков, 10\% молодых людей и 9\% пожилых людей. К этой когорте пациентов может быть применим ингибитор ABL1 - иматиниб, а также двойной ингибитор ABL1/SRC - дазатиниб, способные блокировать передачу сигналов ниже по каскаду за каждым из слитых белков как in vitro, так $и$ in vivo.

Активация сигнального пути JAK-STAT чаще всего является следствием перестроек генов CRLF2 (до 40\% случаев BCR-ABL1-подобного ОЛЛ), EPOR, IL7R, SH2B3 и других. В данном случае наиболее эффективным признан ингибитор JAK1/JAK2 - руксолитиниб [1].

При BCR-ABL1-подобном ОЛЛ известно о других слияниях генов киназ (например, BLNK, NTRK3 и TYK2), которые не поддаются воздействию ингибиторов ни семейства ABL, ни JAK. В данном случае используются энтректиниб и ларотректиниб, которые продемонстрировали эффективность in vitro $и$ in vivo.

Помимо таргетной терапии обнадеживает активность препаратов моноклональных антител, в частности биспецифических Т-клеточных ингибиторов - инотузумаба и блинатумомаба. Применение иммунотерапии на ранних этапах терапии BCR-ABL1-подобного ОЛЛ может привести к улучшению показателей лечения и снижению необходимости ранней аллогенной трансплантации стволовых клеток.

Наконец, CAR-T-терапия также является перспективным методом и может применяться у пациентов с первичным BCR-ABL1-подобным Олл [1].

\section{Разнообразие генетических нарушений при BCR-ABL1- подобном ОЛЛ}

Несмотря на то что BCR-ABL1-подобный ОЛЛ первоначально был идентифицирован по характерной экспрессии генов у пациентов с плохим клиническим исходом [1], не существует ни одного дискретного цитогенетического или молекулярного нарушения, которое бы определяло этот подтип лейкоза $[1,21]$.

При BCR-ABL1-подобном ОЛЛ описано более 70 цитогенетических перестроек, о которых сообщалось в различных исследованиях. Наиболее распространенные из них можно объединить в условные группы по 3'-функциональному партнеру для слияния:

- перестройки с участием генов семейства ABL (ABL1 (Абельсон-киназа 1), ABL2 (Абельсон-киназа 2), CSF1R (рецептор колониестимулирующего фактора 1), PDGFRA и PDGFRB (рецепторы тромбоцитарного фактора роста А и В));

- с сучастием CRLF2 (цитокиновый рецептор-подобный фактор 2);

- с участием EPOR (рецептор эритропоэтина);

- с участием JAK2 (янус-киназа 2). 
Частота указанных перестроек при BCR-ABL1-подобном ОЛЛ незначительно меняется с возрастом, но в различных исследованиях их наличие связано с плохим клиническим исходом. Транслокации из различных групп не являются взаимоисключающими, часто идентифицируют несколько цитогенетических нарушений в одном клиническом случае [21].

Наиболее распространенными нарушениями при BCR-ABL1подобном ОЛЛ являются перестройки с участием гена CRLF2 и его аберрации (до 40\% случаев BCR-ABL1-подобного ОЛЛ). Этот рецептор участвует в раннем развитии В-клеток и активации JAK-STAT сигнального пути, а нарушения функционирования CRLF2 могут быть результатом:

1) перестройки с локусом 14q32 тяжелой цепи иммуноглобулина (IGH), вследствие чего CRLF2 перемещается под контроль энхансера IGH c последующей гиперэкспрессией белка;

2) очаговой делеции в псевдоаутосомной области половых хромосом, приводящей к слиянию P2RY8-CRLF2, что также вызывает гиперэкспрессию белка;

3) реже - активирующей точечной мутации CRLF2 F232C [7, 21].

Преимущественно все перестройки CRLF2 ассоциированы с повышением экспрессии этого белка, но не всегда гиперэкспрессия белка является признаком наличия перестроек: в 20-40\% случаев (в зависимости от группы риска) гиперэкспрессия является следствием дупликации локуса CRLF2, чаще вторичной по отношению к дупликациям хромосом $\mathrm{X}$ или $\mathrm{Y}$ [21].

Среди пациентов высокой группы риска частота перестройки IGHCRLF2 в 2 раза выше, чем P2RY8-CRLF2, и ассоциирована со старшим возрастом, в то время как слияние P2RY8-CRLF2 значительно чаще встречается у детей с соотношением от 2:1 до 5:1 (относительная частота зависит от метода диагностики и выборки пациентов) в некоторых исследованиях [1, 3]. В других исследованиях сообщалось, что P2RY8-CRLF2 встречается чаще, особенно у детей стандартной группы риска, независимо от наличия BCR-ABL1-подобного профиля экспрессии генов [4, 22].

Перестройки JAK2 в результате интерстициальной делеции или реаранжировки с рядом различных генов-партнеров наблюдаются примерно в 5\% BCR-ABL1-подобных ОЛЛ (описано 19 различных химерных слияний генов, среди партнеров наиболее распространены EBF1, ETV6, PAX5 и BCR) без сопутствующих изменений CRLF2. В некоторых исследованиях частота встречаемости таких транслокаций достигает $9 \%$. Частота слияний ЈАК2 одинакова у пациентов всех возрастов [21], хотя в некоторых исследованиях чаще выявлялась у молодых взрослых [23].

Мутации в генах JAK тесно связаны с перестройками CRLF2 при BCR-ABL1-подобном ОЛЛ. Ассоциированные с В-ОЛЛ мутации JAK2 (реже - мутации JAK1) чаще всего происходят в экзоне 16 [21, 23, 24] и отличаются от канонической мутации JAK2 V617F, которая преимущественно встречается у взрослых пациентов с миелопролиферативными новообразованиями. От 40 до 50\% случаев BCR-ABL1-подобного ОЛЛ с перестройками CRLF2 несут патогенные мутации JAK2 (чаще R683G в домене псевдокиназы), которые не выявляются в сочетании с другими аберрациями [1]. Некоторые авторы сообщают, что независимо от перестроек CRLF2 нарушение функций гена JAK2 в результате делеции или 
транслокации с рядом различных генов-партнеров наблюдается примерно в 5-7\% случаев BCR-ABL1-подобного ОЛЛ. Мутации JAK1 менее распространены, но функционально аналогичны. Все эти генетические изменения приводят к активации JAK-STAT пути [3, 21]. Редкие делеции SH2B3 (отрицательный регулятор JAK) и вставки/делеции IL7RA также встречаются при BCR-ABL1-подобном ОЛЛ и приводят к активации передачи сигналов JAK-STAT пути [3]. Соответственно, наличие мутаций в указанных генах у пациентов с перестройками CRLF2 при BCR-ABL1подобном ОЛЛ ассоциируется с худшей выживаемостью [7].

В дополнение к перестройкам CRLF2 и JAK2 конститутивная активация передачи сигналов JAK-STAT может быть результатом перестроек рецептора эритропоэтина (EPOR), наблюдаемых приблизительно у 1-5\% пациентов с BCR-ABL1-подобным ОЛЛ. Наиболее распространенными генами-партнерами являются IGH, IGK, LAIR1 и THADA, хотя встречаются и другие. Эти транслокации приводят к гиперэкспрессии короткой изоформы белка EPOR (в норме вызывает аутофосфорилирование киназы JAK2), утратившего негативно-регуляторный домен, что приводит к активации сигнального пути JAK-STAT и стимулирует лейкемогенез. Наиболее часто встречается инсерция (реже транслокация) гена EPOR в энхансерные области локуса тяжелой (каппа) цепи иммуноглобулина. В отличие от транслокации IGH-EPOR t(14;19)(q32;p13), которую можно обнаружить с помощью FISH, инсерция в настоящее время обнаруживается только с использованием технологий секвенирования нового поколения. Встречаются также инсерции EPOR в верхнюю область гена LAIR1 или локусы гена THADA. Частота перестроек EPOR, по-видимому, наиболее высока у молодых людей (9\%) по сравнению с детьми, подростками и взрослыми пациентами (5, 3 и 1\% соответственно) $[2,7,22,25]$.

Следующая наиболее распространенная группа аберраций, характеризующих BCR-ABL1-подобный ОЛЛ, - слияния с вовлечением ABL1, ABL2, CSF1R и PDGFRB. Частота таких перестроек варьирует от 13 до $19 \%$ всех случаев BCR-ABL1-подобного ОЛЛ и уменьшается с возрастом (16,7\% у детей против 6,5\% у взрослых) [1, 7]. Гены ABL1 и ABL2 представляют собой внутриклеточные киназы со сходной структурой (обычно называемые семейством киназ ABL), которые участвуют в регулировании пролиферации и выживания клеток. В нормальных условиях активация киназ ABL опосредуется несколькими сигналами от рецепторов факторов роста. Геномный анализ образцов пациентов C BCR-ABL1-подобным ОЛЛ выявил множественные транслокации, а также внутрихромосомные делеции и инверсии, активирующие путь передачи сигналов киназ $\mathrm{ABL}[21,26]$. В настоящее время идентифицировано более 20 различных генов-партнеров семейства ABL. Затрагиваются гены $A B L 1 / A B L 2$ и гены рецепторов, экспрессирующихся на поверхности миелоидных клеток, таких как бета-рецептор фактора роста тромбоцитов (PDGFRB) и рецепторы колониестимулирующих факторов (GMSCF/CSF1R) [26]. В результате аберраций продуцируются конститутивно-активные формы белков тирозинкиназ, что приводит к нерегулируемому клеточному делению [21]. Для гена PDGFRB идентифицировано всего 5 генов-партнеров, а именно: EBF1, SSBP2, TNIP1, ZEB2 и ATF7IP, все они встречаются при BCRABL1-подобном ОЛЛ. Перестройка EBF1-PDGFRB чаще всего выявляется 
у пациентов детского возраста (в среднем 12 лет), при этом регистрируются особенно высокий уровень МОБ, плохой ответ на индукционную терапию и худший исход $[7,17,27,28]$.

Особое внимание следует уделить диагностике аберраций транскрипционного фактора IKZF1, которые не являются специфическими для BCR-ABL1-подобного ОЛЛ, но широко распространены при данном подтипе лейкоза: частота внутригенных делеций в среднем составляет $68 \%$ среди пациентов всех возрастов (81\% у взрослых пациентов и $73 \%$ у детей), тогда как среди BCR-ABL1-негативных ОЛЛ частота делеций не превышает 12-15\% [7]. IKZF1 действует как критический регулятор дифференцировки лимфоидных клеток на разных этапах их развития $[29,30]$.

Делеции IKZF1 в 78\% случаев BCR-ABL1-подобного ОЛЛ сочетаются с перестройками генов семейств ABL, CRLF2, EPOR или JAK2 и в 33\% случаев - с мутациями в генах сигнальных путей RAS или JAK-STAT, а также генов факторов развития лимфоидных клеток (ETV6, ERG, PAX5, TCF3 и др.). Пациенты с BCR-ABL1-подобным ОЛЛ при наличии делеций IKZF1 имеют самые худшие показатели выживаемости без событий (EFS) по сравнению с другими группами [29, 30]. На основании обширного исследования группой AIEOP-BFM под руководством M. Stanulla описан особо неблагоприятный генетический профиль - IKZF1 plus. Профиль характеризуется наличием внутригенной делеции IKZF1 в сочетании с делециями 1 или более из следующих генов: CDKN2A, CDKN2B, PAX5, делеций в псевдоаутосомном регионе PAR1 при отсутствии делеции ERG [30, 31]. Для пациентов IKZF1 ${ }^{\text {plus }}$ характерны значимо худшие показатели EFS, чем для пациентов с 1 делецией IKZF1 или IKZF1 дикого типа $(p=0,02)$.

Функция гена IKZF1 также может нарушаться при инсерциях, сдвигах рамки считывания, миссенс-мутациях, которые наблюдаются примерно в 7\% случаев В-линейных ОЛЛ [29, 30], и редкими слияниями (IKZF1NUTM1 и IKZF1-SETD5). Однако патогенетическая роль этих аберраций в развитии ОЛЛ до настоящего времени не установлена [30].

Безусловно, при BCR-ABL1-подобном ОЛЛ выявляются и другие аберрации. Так, редкие слияния киназ с участием генов NTRK3, BLNK, DGKH, PTK2B, FLT3, FGFR1, TYK2 суммарно встречаются примерно в 5\% BCR-ABL1-подобного ОЛЛ [7, 8]. Сообщается и о других редких изменениях (2,4\%), ассоциированных с BCR-ABL1-подобным ОЛЛ, например о делециях SH2B3 [1]. Еще одна необычная транслокация ETV6-NTRK3 была идентифицирована в 1\% случаев BCR-ABL1-подобного ОЛЛ. Однако такие слияния были выявлены при ряде других злокачественных новообразований, включая карциному молочной железы, саркому, острый миелоидный лейкоз и глиому [32].

Нарушения активации пути JAK-STAT иногда могут быть вызваны не перестройками ключевых генов цепи, а делециями генов JAK1, JAK3, IL7R, SH2B3, IL2RB или TYK2 [7]. Как правило, эти делеции являются мульти-/субклональными вторичными событиями лейкемогенеза, возникающими после основной хромосомной перестройки [2].

Мутации ZNF483, связанные с BCR-ABL1-подобным ОЛЛ, являются новыми и встречаются только при наличии изменений в гене IKZF1 [33].

Мутации в генах NRAS, KRAS, PTPN11, NF1, BRAF и FLT3 были обнаружены у небольшого числа пациентов с BCR-ABL1-подобным ОлЛ [1, 
34], но встречаются также при других подтипах ОЛЛ [8]. Многие из этих (обычно субклональных) мутаций пути RAS возникают одновременно с BCR-ABL1-подобными транслокациями, например слияниями генов ABL, CRLF2, EPOR или JAK2 [1, 33], в то время как другие мутации происходят изолированно [8]. По данным некоторых исследований, встречаемость мутаций пути RAS составляет 3,6\% [35]. В других исследованиях показано, что частота мутаций в генах NRAS, KRAS, PTPN11, NF1 и BRAF может достигать 5\% от всех случаев BCR-ABL1-подобного ОЛЛ [7]. В одном исследовании 3,9\% пациентов с BCR-ABL1-подобным ОЛЛ имели только активирующие мутации FLT3. Однако оценка действия этих редких мутаций на исход терапии и их влияние на выживаемость не исследованы в связи с низкой встречаемостью [15].

\section{Диагностика BCR-ABL1-подобного ОЛЛ}

Несмотря на то что в последние годы проведено немало попыток структурировать генетические нарушения для диагностики BCR-ABL1подобного ОЛЛ, эта проблема до сих пор на пике актуальности во всем мире. Стратегии, используемые для определения BCR-ABL1-подобного ОЛЛ, различаются, однако общая цель диагностического тестирования заключается в точной идентификации основных генетических изменений, чтобы соответствующим образом скорректировать лечение.

Диагностика BCR-ABL1-подобного ОЛЛ проводится для всех первичных пациентов из группы «других В-линейных ОЛЛ» («B-other»), что подразумевает отсутствие у пациента неслучайных количественных и структурных аберраций, таких как гипердиплоидный $(\mathrm{n}=51-65)$ или гиподиплоидный $(\mathrm{n}<45)$ кариотип, перестройки с участием гена $\mathrm{MLL}$, транслокации BCR-ABL1, ETV6-RUNX1, TCF3-PBX1, а также iAMP21 (внутрихромосомная амплификация 21q). Перечисленные аберрации не исключают наличия BCR-ABL1-подобного ОЛЛ, но характеризуются высокой распространенностью при ОЛЛ, хорошо изучены в отношении прогноза заболевания и чаще всего определяют стратегию его лечения, в связи с чем диагностика BCR-ABL1-подобного ОЛЛ в этой группе пациентов нецелесообразна при наличии адекватного ответа на терапию у таких пациентов.

В группу «других В-линейных ОЛЛ» попадают до 35\% случаев первичных ОЛЛ среди детей - именно эти пациенты представляют особый интерес для поиска дополнительных прогностических аберраций, в том числе ассоциированных с BCR-ABL1-подобным ОЛЛ. Для достижения этих целей в настоящее время используются описанные ниже подходы и их комбинации, а универсального алгоритма диагностики не существует.

\section{Методы диагностики BCR-ABL1-подобного ОЛЛ}

Профилирование экспрессии генов (GEP) чаще всего методом анализа микрочипов низкой плотности на основе технологии TaqMan (LDA) является наиболее распространенным для идентификации BCR-ABL1подобного ОЛЛ. Данные по экспрессии генов подвергаются математической обработке и классификации. Первые классификаторы были разработаны более 10 лет назад группами под руководством M. den Boer и Ch. Mullighan. Данные по экспрессии генов были получены методом LDA 
и обработаны математическими алгоритмами иерархической кластеризации данных (НС) или предсказательного анализа микрочипов (РАМ) [1, 10]. Результаты работы 2 групп дали схожие результаты при диагностике образцов. Однако следует учитывать, что 2 разные исследовательские группы идентифицировали BCR-ABL1-подобный ОЛЛ, используя разные и только частично перекрывающиеся наборы зондов [21].

B настоящее время для диагностики BCR-ABL1-подобного ОЛЛ в некоторых клинических лабораториях на первом этапе используют LDA в дополнение к стандартному морфологическому анализу, иммунофенотипированию, кариотипированию и FISH. Такой подход в основном применяется для педиатрических пациентов высокого риска (NCI) [1, 36].

LDA является эффективным инструментом скрининга, но выгодным при большом количестве пациентов в многоцентровых исследованиях и чаще всего используется в рамках клинических испытаний. Результаты LDA получают в течение 48 часов с момента взятия биологического образца, что позволяет быстро и эффективно скорректировать лечение пациентов [1, 10]. Более доступной альтернативой LDA может стать использование количественной полимеразной цепной реакции в реальном времени (ОТ-ПЦР) для анализа экспрессии единичных генов, свойственных BCR-ABL1-подобному фенотипу ОЛЛ.

Дальнейший алгоритм диагностики определяется результатами, полученными при анализе экспрессии генов. Так, в случае гиперэкспрессии CRLF2, по данным LDA, проводят флуоресцентную гибридизацию in situ (FISH) для идентификации реаранжировок CRLF2. Затем осуществляют мутационное тестирование JAK1 и JAK2 у всех пациентов с подтвержденными реаранжировками CRLF2. У пациентов без реаранжировок CRLF2 проводят OT-ПЦР-анализ для выявления реаранжировок ABL, JAK2 и других известных киназ, подтверждая результаты ПЦР секвенированием по Сэнгеру. Наконец, осуществляют секвенирование PHK оставшихся LDA-положительных случаев ОЛЛ без выявленных генетически изменений. Этот алгоритм в настоящее время используется в клиническом испытании COG AALL1131 фаза 3 (NCT02883049). Другие группы исследователей используют аналогичные или сокращенные алгоритмы диагностики (табл. 3).

Реаранжировки ABL1, ABL2, JAK2, PDGFRB, CSF1R и EPOR, типичные для BCR-ABL1-подобного ОЛЛ, также могут быть идентифицированы с помощью FISH. Разработаны разрывные зонды, используемые в рутинной клинической практике. Однако применение FISH как единственного диагностического метода позволяет идентифицировать не более 75\% случаев BCR-ABL1-подобного ОЛЛ, поскольку обнаруживает лишь ограниченное число специфических транслокаций $[1,21]$. Также при FISH часто невозможно идентифицировать 5'-партнера слияния генов, поэтому в некоторых случаях требуется дополнительная молекулярная диагностика для подтверждения тех или иных аберраций [1]. В этом контексте нельзя не упомянуть метод проточной цитометрии для оценки поверхностной экспрессии CRLF2 [1, 21]. Клиническое иммунофенотипирование CRLF2 является высокоэффективным и позволяет идентифицировать пациентов с вероятными реаранжировками CRLF2 в течение нескольких часов после получения образца. Однако чувствительность гиперэкспрессии CRLF2 для обнаружения BCR-ABL1-подобного ОЛЛ 


\section{Таблица 3}

Методы диагностики BCR-ABL1-подобного ОлЛ в различных исследованиях

\begin{tabular}{|c|c|c|c|c|}
\hline $\begin{array}{l}\text { Автор (год); тип } \\
\text { исследования } \\
\text { (период) }\end{array}$ & $\begin{array}{l}\text { Кол-во пациентов } \\
\text { с В-ОЛЛ }\end{array}$ & $\begin{array}{l}\text { Частота } \\
\text { BCR-ABL1- } \\
\text { подобного ОлЛ, } \\
\text { чел. (\%) }\end{array}$ & $\begin{array}{l}\text { Возраст (медиа- } \\
\text { на, лет) }\end{array}$ & $\begin{array}{l}\text { Использованные } \\
\text { методы диагно- } \\
\text { стики BCR-ABL1- } \\
\text { подобного ОЛЛ }\end{array}$ \\
\hline $\begin{array}{l}\text { Den Boer и соавт. } \\
\text { (2009); } \\
\text { проспективное } \\
(1990-2004)\end{array}$ & 246 & $44(17,9 \%)$ & 6,5 & $\begin{array}{l}\text { GEP (HP) } \\
\text { ОТ-ПЦР }\end{array}$ \\
\hline $\begin{array}{l}\text { Harvey и соавт. } \\
(2010) ; \\
\text { ретроспективное } \\
(2000-2003)\end{array}$ & 207 & $29(14,0 \%)$ & 14,2 & $\begin{array}{l}\text { GEP (PAM), } \\
\text { FISH, проточная } \\
\text { цитометрия, ПЦР }\end{array}$ \\
\hline $\begin{array}{l}\text { Roberts и соавт. } \\
\text { (2014); } \\
\text { проспективное } \\
\text { (2000-2007) }\end{array}$ & 344 & $40(11,6 \%)$ & 5,3 & $\begin{array}{l}\text { GEP (PAM), } \\
\text { FISH, ОT-ПЦР, сек- } \\
\text { венирование PHK, } \\
\text { секвенирование } \\
\text { по Сэнгеру, NGS, } \\
\text { геномная ПЦР }\end{array}$ \\
\hline $\begin{array}{l}\text { lacobucсі и соавт. } \\
\text { (2016); } \\
\text { ретроспективное }\end{array}$ & 3115 & $212(6,8 \%)$ & - & $\begin{array}{l}\text { Секвенирование } \\
\text { по Сэнгеру, полно- } \\
\text { геномное секвени- } \\
\text { рование }\end{array}$ \\
\hline $\begin{array}{l}\text { Konoplev и соавт. } \\
\text { (2017); } \\
\text { проспективное } \\
(2013-2015)\end{array}$ & 126 & $10(7,9 \%)$ & - & $\begin{array}{l}\text { Мультипараметри- } \\
\text { ческая проточная } \\
\text { цитометрия, FISH }\end{array}$ \\
\hline $\begin{array}{l}\text { Tasian и соавт. } \\
\text { (2017); } \\
\text { ретроспективное }\end{array}$ & 87 & $18(20,7 \%)$ & 28,6 & $\begin{array}{l}\text { GEP (PAM), } \\
\text { FISH, ОТ-ПЦР }\end{array}$ \\
\hline $\begin{array}{l}\text { Herold и соавт. } \\
\text { (2017); } \\
\text { ретроспективное } \\
(1999-2005)\end{array}$ & 207 & $26(12,6 \%)$ & 31 & $\begin{array}{l}\text { GEP (PAM), } \\
\text { FISH, OT-ПЦР, MLPA }\end{array}$ \\
\hline $\begin{array}{l}\text { Roberts и соавт. } \\
\text { (2017); } \\
\text { ретроспективное }\end{array}$ & 798 & $194(24,3 \%)$ & 40 & $\begin{array}{l}\text { GEP (PAM), } \\
\text { FISH, оценка коли- } \\
\text { чества копий ДНК, } \\
\text { анализ SNP }\end{array}$ \\
\hline $\begin{array}{l}\text { Reshmi и соавт. } \\
\text { (2017); } \\
\text { ретроспективное } \\
(2010-2014)\end{array}$ & 1389 & $284(20,4 \%)$ & - & $\begin{array}{l}\text { GEP, OT-ПЦР, секве- } \\
\text { нирование транс- } \\
\text { криптома, FISH }\end{array}$ \\
\hline $\begin{array}{l}\text { Roberts и соавт. } \\
\text { (2018); } \\
\text { ретроспективное } \\
(2006-2008)\end{array}$ & 1023 & $139(13,6 \%)$ & $<10$ & $\begin{array}{l}\text { GEP (8 генов) } \\
\text { FISH, секвениро- } \\
\text { вание по Сэнгеру, } \\
\text { OT-ПЦР, секвениро- } \\
\text { вание транскрип- } \\
\text { тома }\end{array}$ \\
\hline
\end{tabular}

относительно ограниченна, с учетом того что реаранжировки CRLF2 наблюдаются только в 50\% случаев всех BCR-ABL1-подобных ОЛЛ, а гиперэкспрессия CRLF2 не всегда свидетельствует о наличии реаранжировки. Чувствительность метода в отношении реаранжировок CRLF2 составляет 92\% [21]. Другими словами, для подтверждения реаранжировки по возможности следует прибегнуть к FISH и/или ОТ-ПЦР. 
Table 3

Diagnostic methods of BCR-ABL1-like ALL used in various studies

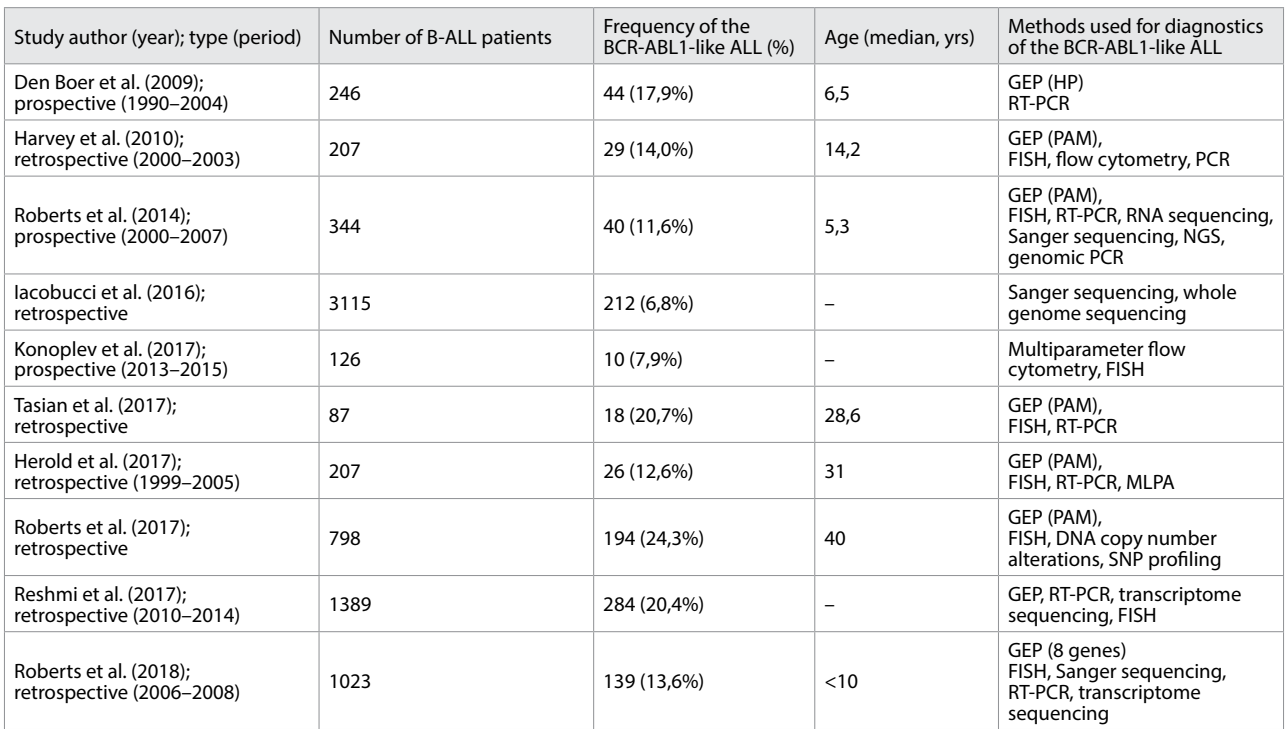

В дополнение к вышеупомянутым методам, используемым в настоящее время эталонными лабораториями COG [10], несколько коммерчески доступных платформ для секвенирования нового поколения (NGS) также могут использоваться для идентификации BCR-ABL1-подобных изменений при ОЛЛ. Например, панель FoundationOne Heme представляет собой целевой комбинированный метод секвенирования PHК и ДНК, способный обнаруживать слияния и мутации в более чем 400 генах, а панель NanoString nCounter позволяет идентифицировать более 200 известных онкогенных слияний, ассоциированных с лейкозами [36]. Коммерческие платформы, так же как и секвенирование всего генома (WGS), экзома (WES), транскриптома (RNA-seq), в настоящее время применяются только на уровне исследований. Преимуществом таких подходов является обнаружение новых изоформ сплайсинга и слияний генов, которые могут быть упущены целевыми методами. Секвенирование идеально подходит для углубленного анализа случаев BCR-ABL1подобного ОЛЛ, но в настоящее время не годится в качестве широко распространенного метода скрининга. Тем не менее секвенирование становится все более доступным и, вероятно, в будущем дополнит или заменит существующие диагностические целевые платформы, хотя этот подход остается довольно дорогостоящим и характеризуется относительно долгим временем выполнения, а также требует комплексного биоинформатического анализа.

Таким образом, структура молекулярно-генетических изменений при BCR-ABL1-подобном ОЛЛ настолько сложна, что наиболее актуальным в настоящее время остается вопрос поиска четких молекулярнобиологических критериев стратификации таких пациентов, дифференциальной диагностики BCR-ABL1-подобного ОЛЛ с другими BCR-ABL1негативными ОЛЛ, усовершенствования и адаптации молекулярно-био- 
логических методов диагностики BCR-ABL1-подобного ОЛЛ для рутинной лабораторной диагностики.

Вклад авторов: концепция, сбор и обработка информации, написание текста, редактирование - Вшивкова О.С.; сбор и обработка информации, написание текста - Корзик А.В.

Authors' contribution: concept, collection and processing of information, text writing, editing - O.S. Vshivkova; collection and processing of information, writing text - Korzik A.V.

Конфликт интересов. Авторы заявляют об отсутствии конфликта интересов.

Conflict of interest. The authors declare no conflict of interest.

\section{- ЛИТЕPATYPA/REFERENCES}

1. Harvey R.C., Tasian S.K. (2020) Clinical diagnostics and treatment strategies for Philadelphia chromosome-like acute lymphoblastic leukemia. Blood Adv, vol. 4, no 1, pp. 218-228.

2. Roberts K.G. (2017) The biology of Philadelphia chromosome-like ALL. Best Pract Res Clin Haematol, vol. 30, no 3, pp. $212-221$.

3. Tasian S.K., Loh L.M., Hunger S.P. (2017) Philadelphia chromosome-like acute lymphoblastic leukemia. Blood, vol. 130, no 19, pp. 2064-2072.

4. Boer J.M., Marchante J.R., Evans W.E. (2015) BCR-ABL1-like cases in pediatric acute lymphoblastic leukemia: a comparison between DCOG/ Erasmus MC and COG/St. Jude signatures. Haematol Rep, vol. 100, no 9, pp. 354-7.

5. Gluzman D.F., Sklyarenko L.M., Ivanivskaya T.S. (2016) Updated world health organization classification of myeloid neoplasms and acute leukemias (2016 revision). Onkologiya, vol. 18, pp. 184-191.

6. Arber D.A., Orazi A., Hasserjian R. (2016) The 2016 revision to the World Health Organization classification of myeloid neoplasms and acute leukemia. Blood, vol. 127, no 20, pp. 2391-405.

7. Khan M., Siddiqi R., Tran T.H. (2018) Philadelphia chromosome-like acute lymphoblastic leukemia: A review of the genetic basis, clinical features, and therapeutic options. Semin Hematol, vol. 55, no 4, pp. 235-241.

8. Tran T.H., Loh M.L. (2016) Ph-like acute lymphoblastic leukemia. Hematology Am Soc Hematol Educ Program, vol. 2016, no 1, pp. 561-566.

9. Herold T., Baldus C.D., Gokbuget N. (2014) Ph-like acute lymphoblastic leukemia in older adults. N Engl J Med, vol. 371 , no 23, p. 2235.

10. Jain N., Roberts K.G., Jabbour E. (2017) Ph-like acute lymphoblastic leukemia: a high-risk subtype in adults. Blood, vol. 129 , pp. 572-81.

11. Boer J.M., Koenders J.E., van der Holt B. (2015) Expression profiling of adult acute lymphoblastic leukemia identifies a BCR-ABL1-like subgroup characterized by high non-response and relapse rates. Haematol Rep, vol. 100, no 7, pp. 261-264.

12. Roberts K.G., Gu Z., Payne-Turner D. (2017) High frequency and poor outcome of Philadelphia chromosome-like acute lymphoblastic leukemia in adults. J Clin Oncol, vol. 35, pp. 394-401.

13. Konoplev S., Lu X., Konopleva M. (2017) CRLF2-Positive B-Cell Acute Lymphoblastic Leukemia in Adult Patients: A Single-Institution Experience, Am J Clin Pathol, vol. 147, no 4, pp. 357-363.

14. Loh M.L., Zhang J., Harvey R.C. (2013) Tyrosine kinome sequencing of pediatric acute lymphoblastic leukemia: a report from the Children's Oncology Group TARGET Project. Blood, vol. 121, no 3, pp. 485-488.

15. Reshmi S.C., Harvey R.C., Roberts K.G. (2017) Targetable kinase gene fusions in high-risk B-ALL: a study from the Children's Oncology Group. Blood, vol. 129, pp. 3352-61.

16. Zaliova M., Stuchly J., Winkowska L. (2019) Genomic landscape of pediatric B-other acute lymphoblastic leukemia in a consecutive European cohort. Haematol Rep, vol. 104, no 7, pp. 1396-1406.

17. Schwab C., Ryan S.L., Chilton L. (2016) EBF1-PDGFRB fusion in pediatric B-cell precursor acute lymphoblastic leukemia (BCP-ALL): genetic profile and clinical implications. Blood, vol. 127, no 18, pp. 2214-2218

18. Roberts K.G., Pei D., Campana D. (2014) Outcomes of children with BCR-ABL1-like acute lymphoblastic leukemia treated with risk-directed therapy based on the levels of minimal residual disease. J Clin Oncol, vol. 32, pp. 3012-20.

19. Herold T., Schneider S., Metzeler K.H. (2017) Adults with Philadelphia chromosome-like acute lymphoblastic leukemia frequently have IGHCRLF2 and JAK2 mutations, persistence of minimal residual disease and poor prognosis. Haematol Rep, vol. 102, no 1, pp. 130-138.

20. Den Boer M.L., van Slegtenhorst M., De Menezes R.X. (2009) A subtype of childhood acute lymphoblastic leukaemia with poor treatment outcome: a genome-wide classification study. Lancet Oncol, vol. 10, no 2, pp. 125-134.

21. Conant J.L., Czuchlewski D.R. (2019) BCR-ABL1-like B-lymphoblastic leukemia/lymphoma: Review of the entity and detection methodologies. Int J Lab Hematol, vol. 41, pp. 126-130.

22. Ensor H.M., Schwab C., Russell L.J. (2011) Demographic, clinical, and outcome features of children with acute lymphoblastic leukemia and CRLF2 deregulation: results from the MRC ALL97 clinical trial. Blood, vol. 117, no 7, pp. 2129-2136.

23. Harvey R.C., Tasian S.K. (2020) Clinical diagnostics and treatment strategies for Philadelphia chromosome-like acute lymphoblastic leukemia. Blood Adv, vol. 4, no 1, pp. 218-228.

24. Roberts K.G., Yang Y.L., Payne-Turner D. (2017) Oncogenic role and therapeutic targeting of ABL-class and JAK-STAT activating kinase alterations in Ph-like ALL. Blood Adv, vol. 1, no 20, pp. 1657-1671.

25. Iacobucci I., Li Y., Roberts K.G. (2016) Truncating Erythropoietin Receptor Rearrangements in Acute Lymphoblastic Leukemia. Cancer Cell, vol. 29, pp. 186-200. 
26. Weston B.W., Hayden M.A., Roberts K.G. (2013) Tyrosine kinase inhibitor therapy induces remission in a patient with refractory EBF1-PDGFRBpositive acute lymphoblastic leukemia. J Clin Oncol, vol. 31, no 25, pp. 413-416.

27. Imamura T., Kiyokawa N., Kato M. (2016) Characterization of pediatric Philadelphia-negative B-cell precursor acute lymphoblastic leukemia with kinase fusions in Japan. Blood Cancer, vol. 6, no 5, p. 419.

28. Tsaur G.A., Olshanskaya Yu.V., Druy A.E. (2019) BCR-ABL1-podobnyj ostryj limfoblastnyj lejkoz u detej [BCR-ABLI-like pediatric acute lymphoblastic leukemia]. Pediatric Hematology/Oncology and Immunopathology, vol. 18, no 1, pp. 112-126.

29. Vshyukova V.S., Meleshko A.N. (2015) Rol' transkripcionnogo faktora Ikaros v normal'nom gemopoeze i lejkozogeneze: biologicheskie i klinicheskie aspekty [The role of Ikaros transcriptional factor in normal hematopoiesis and leukemogenesis: biological and clinical aspects]. Advances in Molecular Oncology, vol. 2, no 1, pp. 013-026.

30. Marke R., van Leeuwen F.N., Scheijen B. (2018) The many faces of IKZF1 in B-cell precursor acute lymphoblastic leukemia. Haematologica, vol. 103, no 4, pp. 565-574.

31. Stanulla M., Dagdan E., Zaliova M. (2018) IKZF1 plus Defines a New Minimal Residual Disease-Dependent Very-Poor Prognostic Profile in Pediatric B-Cell Precursor Acute Lymphoblastic Leukemia. J Clin Oncol, vol. 36, no 12, pp. 1240-1249.

32. Roberts K.G. (2018) Why and how to treat Ph-like ALL? Best Pract Res Clin Haematol, vol. 31, no 4, pp. 351-356.

33. Schroeder M.P., Bastian L., Eckert C. (2019) Integrated analysis of relapsed B-cell precursor Acute Lymphoblastic Leukemia identifies subtypespecific cytokine and metabolic signatures. Sci Rep 9 (electronic journal), article number: 4188. Available at: https://www.nature.com/articles/ s41598-019-40786-1.pdf (accessed November 26, 2020).

34. Roberts K.G., Reshmi S.C., Harvey R.C. (2018) Genomic and outcome analyses of Ph-like ALL in NCl standard-risk patients: a report from the Children's Oncology Group. Blood, vol. 132, no 8, pp. 815-824.

35. Anagnostou T., Knudson R.A., Pearce K.E. (2020) Clinical utility of fluorescence in situ hybridization-based diagnosis of BCR-ABL1 like (Philadelphia chromosome like) B-acute lymphoblastic leukemia. Am J Hematol, vol. 95, no 3, pp. 68-72.

36. Maese L., Tasian S.K., Raetz E.A. (2017) How is the Ph-like signature being incorporated into ALL therapy? Best Pract Res Clin Haematol, vol. 30, no 3, pp. 222-228.

Подана/Submitted: 27.04.2021

Принята/Accepted: 18.05.2021

Контакты/Contacts: e-mail:vshyukova@gmail.com 